\title{
Stereo Sound Board for Real Time Auditory Coding of Visual Information
}

\author{
C. Faik ${ }^{1}$, C. Capelle ${ }^{1 \& 2}$, C. Halet $^{2}$, C. Trullemans ${ }^{2}$, C. Veraart ${ }^{1}$ \\ ${ }^{1}$ Neural Rehab. Engng Lab., Catholic University of Louvain, UCL 54.46, 54 av. Hippocrate, B-1200 Brussels, Belgium. \\ ${ }^{2}$ Microelectronic Lab., Catholic University of Louvain, 3 place du Levant, B-1348 Louvain-La-Neuve, Belgium
}

This paper deals with the design of a stereo sound board for rehabilitation of blindness following the principles of sensory substitution. In this method, signals coding the lacking visual information are transmitted to an intact sensory organ. Moreover, real time functioning of the sensory substitution device has to be attempted to favor sensory motor interactions beneficial to the learning process. In the present study, we consider the feasibility of this conceptual model of sensory substitution and its implementation as a rough model of the retina connected to an inverse model of the cochlea.

The model of vision is limited to main features of the primary visual system, i.e. lateral inhibition and graded resolution. Lateral inhibition can be stimulated by filtering the initial image with an edge detection filter. Graded resolution is modeled using a multi-resolution artificial retina built on the filtered image. The inverse model of audition achieves a weighted summation of sinusoids (i.e. an inverse Fourier transform) whose amplitudes and phases depend on the corresponding ear, taking into account spectral sensitivity of the human ear. Coupling between the model of vision and the inverse model of audition consists of the association of a specific sinusoid to each pixel of the artificial retina; amplitude of each sinusoid is modulated by the gray level of the corresponding pixel.

A specific PC-plugged printed card board has been developed to meet follow the auditory specifications required by the prosthesis: the production of a weighted sum of 256 sinusoids whose phases and frequencies are loaded at card initialization, and whose amplitudes can be modified in real time. This complex sound has to be stereophonic, with different phases and amplitudes for the left and right signals. At this time, no low cost music generator meets these specifications. However, sound processors approach them more and more. Such a processor, the SAM8905 (DREAM s.a. manufacturer), can produce up to 64 monoral sinusoids. Hence, the printed card board contains eight SAM8905's (allowing the generation of 256 binaural sinusoids). The serial outputs of the SAM8905's are converted in parallel by serial-to-parallel shift-registers; then, they are added together. These shift registers and parallel adders are implemented in a FPGA which is followed by two Digital-to-Analog Converters. A control circuitry drives the whole data-path.

The conceptual model of sensory substitution of vision by audition has been implemented in a real time functioning experimental prototype, using this stereo sound board. This prototype is based on a Personal Computer which is connected to a miniature head-fixed video camera on the one hand, and to headphones on the other hand. In a second application, called acoustic screen, this sound board is used in an aid device for blind persons using computers with graphical user interface (Macintosh, Windows, etc.). This device transforms into sound an image which is captured on the computer screen, around the mouse. 
The sound board: description and principle
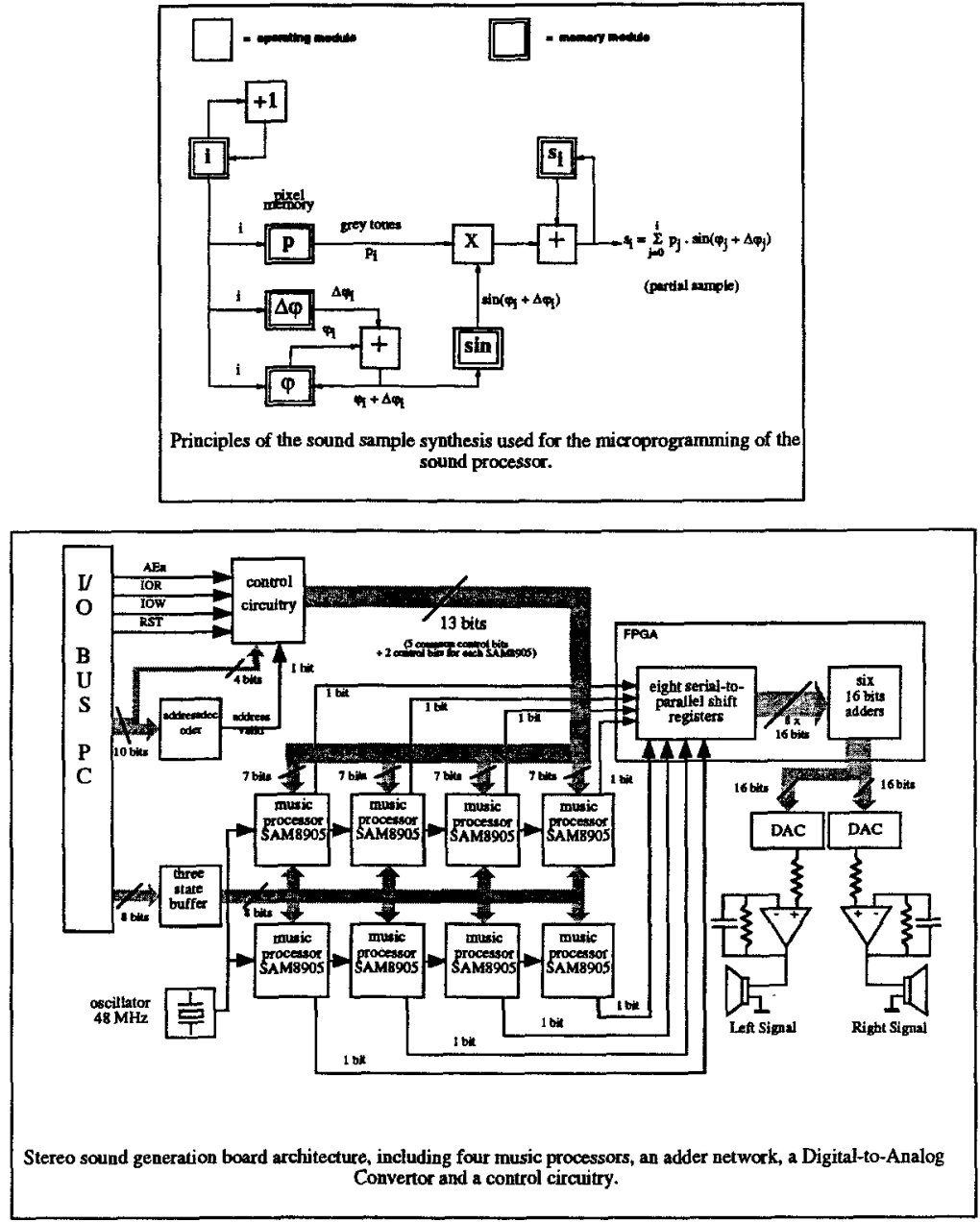

\section{Applications of the sound board:}

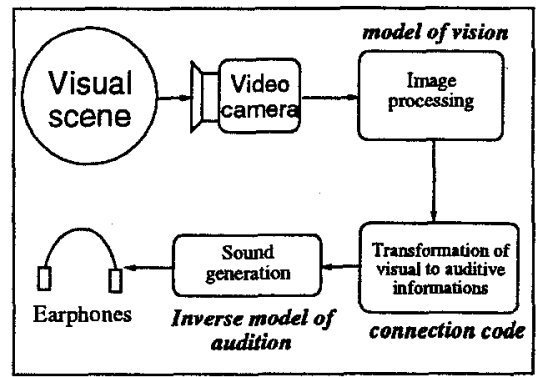

Visual prothesis of sensory substitution

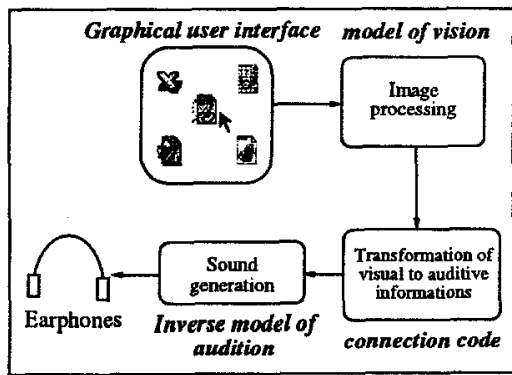

Acoustic screen for computers with graphical interface 